\title{
Observation of Kinetic Plasma Jets in a Coronal-Loop Simulation Experiment
}

\author{
S. K. P. Tripathi, ${ }^{*}$ P. M. Bellan, and G. S. Yun \\ Applied Physics, California Institute of Technology, Pasadena, California 91125, USA \\ (Received 18 November 2005; revised manuscript received 3 June 2006; published 30 March 2007)

\begin{abstract}
Under certain conditions an intense kinetic plasma jet is observed to emerge from the apex of laboratory simulations of coronal plasma loops. Analytic and numerical models show that these jets result from a particle orbit instability in a helical magnetic field whereby magnetic forces radially eject rather than confine ions with sufficiently large countercurrent axial velocity.
\end{abstract}

PACS numbers: 52.72.+v, 52.55.Fa, 52.55.Ip, 96.60.pf

Many lab and space plasmas (e.g., solar coronal loops [1], spheromaks [2], tokamaks [3], and magnetic clouds [4]) are presumed to be magnetic flux tubes filled with plasma confined via magnetohydrodynamic (MHD) forces. However, confinement can be significantly degraded in ways not predicted by MHD; e.g., in tokamaks, ions resulting from neutral beams injected against the toroidal current direction (counterinjection) exhibit severe orbit losses compared to coinjection [5-7]. Related confinement degradation may be the cause of small solar corona jets (e.g., surges) associated with canceling magnetic features [8-10] and of coronal streamers emanating from magnetic neutral lines [11]. This Letter reports that in certain circumstances an ion injected along the axis of a magnetic flux tube will be magnetically ejected from the flux tube instead of being magnetically confined; i.e., the ion will have radially unstable motion (RUM). This instability explains the severe orbit losses of counterinjected ions in tokamaks and is likely relevant to similar situations occurring in the solar corona. The instability, labeled as "kinetic plasma jet" in Fig. 1, was discovered experimentally and then modeled.

We first outline the physical basis for RUM. Consider a particle injected with velocity $v_{z 0}$ near the axis of a cylindrical flux tube having helical magnetic field $\mathbf{B}=B_{\phi} \hat{\phi}+$ $B_{z} \hat{z}$. The flux tube geometry is sketched in Fig. 2(a) and corresponds to a straightened-out model of Fig. 2(b), our laboratory configuration simulating a coronal loop [12]. The $r$ component of $m d \mathbf{v} / d t=q \mathbf{v} \times \mathbf{B}$ is $m \ddot{r}-m r \dot{\phi}^{2}=$ $q r \dot{\phi} B_{z}-q v_{z} B_{\phi}$. If $v_{z} B_{\phi}=0$, then radial force-balance $\ddot{r}=0$ gives $\dot{\phi}=-q B_{z} / m=-\omega_{c}$, i.e., the conventional cyclotron orbit. However, if $v_{z} B_{\phi} \neq 0$, then radial forcebalance $\ddot{r}=0$ requires $\dot{\phi}^{2}+\dot{\phi} q B_{z} / m-v_{z} q B_{\phi} / m r=0$ so no real $\dot{\phi}$ solutions exist if

$$
B_{z}^{2}+4 m v_{z} B_{\phi} / q r<0 .
$$

Thus, large negative $m v_{z} B_{\phi} / q r$ causes the radially outward force $-q v_{z} B_{\phi}$ to overwhelm the radially inward force $q r \dot{\phi} B_{z}$ so centrifugal force $m r \dot{\phi}^{2}$ is unbalanced.

We next use Hamiltonian arguments to show that satisfying Eq. (1) leads to the particle being radially ejected from the flux tube, i.e., RUM. To model the simplest nontrivial situation, both $B_{z}$ and the axial current density $J_{z}$ are assumed uniform within the flux tube so in the flux tube the vector potential is $\mathbf{A}(r)=\hat{\phi} B_{z} r / 2-\hat{z} \mu_{0} J_{z} r^{2} / 4$, the axial flux is $\Phi=B_{z} \pi r^{2}$, and the axial current is $I=J_{z} \pi r^{2}$. Using the Lagrangian $L=m\left(v_{r}^{2}+r^{2} \dot{\phi}^{2}+v_{z}^{2}\right) / 2+$ $q r \dot{\phi} A_{\phi}+q v_{z} A_{z}$, the canonical momenta $P_{\phi}=\partial L / \partial \dot{\phi}$ and $P_{z}=\partial L / \partial v_{z}$ are

$$
P_{\phi}=m r^{2} \dot{\phi}+q r^{2} B_{z} / 2, \quad P_{z}=m v_{z}-\mu_{0} q J_{z} r^{2} / 4
$$

Because $\phi$ and $z$ are ignorable, both $P_{\phi}$ and $P_{z}$ are invariants. A particle injected with velocity $v_{z 0}$ along the flux tube axis (i.e., at $r=0$ ) thus has the invariants

$$
P_{\phi}=0, \quad P_{z}=m v_{z 0}
$$

Combining Eqs. (2) and (3) gives $\dot{\phi}=-\omega_{c} / 2$ and $v_{z}=$ $v_{z 0}+\omega_{c} r^{2} \lambda / 4$ where $\lambda=\mu_{0} J_{z} / B_{z}=\mu_{0} I / \Phi$ is related to twist. The Hamiltonian $H=m\left(v_{r}^{2}+r^{2} \dot{\phi}^{2}+v_{z}^{2}\right) / 2$ can be expressed as $H=m v_{r}^{2} / 2+f(r)$ where

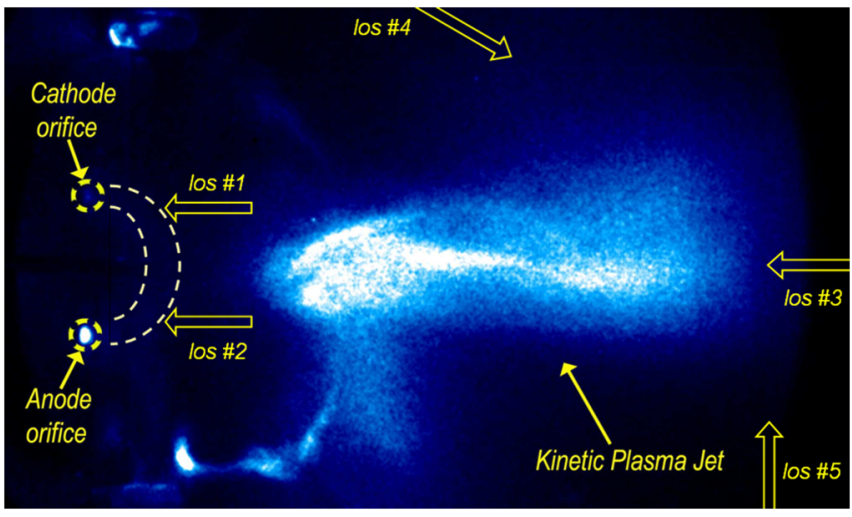

FIG. 1 (color online). Kinetic plasma jet emanating from an argon laboratory loop. Dashed arch corresponds to initial plasma loop at $t \approx 1.0 \mu \mathrm{s}$ as in Fig. 4(b). Arrows represent lines of sight (los \#) used for spectroscopy; arrow widths represent $\sim 6 \mathrm{~mm}$ diameter of lines of sight. 
(a)

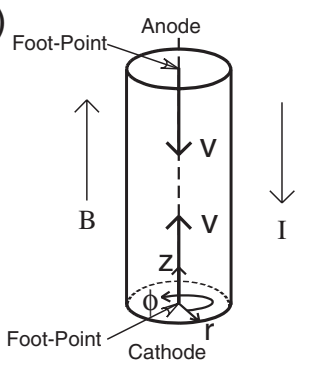

(b)

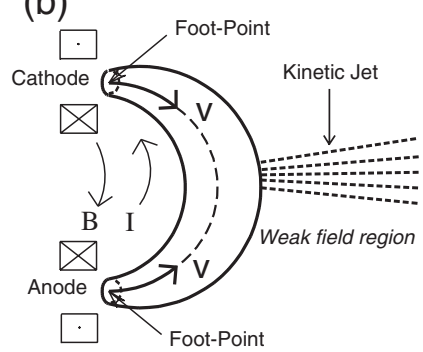

FIG. 2. (a) Flux tube geometry used in the model. (b) Experimental configuration; $B$ outside the flux loop is weakest on the right-hand side (indicated as weak field region).

$$
f(r)=\frac{m \omega_{c}^{2}}{2 \lambda^{2}}\left[\frac{\lambda^{2} r^{2}}{4}+\left(\frac{\lambda v_{z 0}}{\omega_{c}}+\frac{\lambda^{2} r^{2}}{4}\right)^{2}\right]
$$

is an effective potential. On defining $\xi=|\lambda| r$, the dimensionless effective potential $V(\xi)=2 \lambda^{2} f(r) / m \omega_{c}^{2}$ can be written as

$$
V(\xi)=\xi^{2} / 4+\left(\lambda v_{z 0} / \omega_{c}+\xi^{2} / 4\right)^{2} .
$$

Equation (5) gives $\partial V / \partial \xi=\xi / 2+\lambda v_{z 0} \xi / \omega_{c}+\xi^{3} / 4$. Near the flux tube axis the $\xi^{3} / 4$ term is negligible, so negative $\partial V / \partial \xi$ near the axis corresponds to having

$$
S=\lambda v_{z 0} / \omega_{c}+0.5<0 .
$$

Our main result is that if $S<0$ so $\partial V / \partial \xi<0$ near the axis, a particle at $r=0$ is on an effective potential hill as shown in the $S=-0.5$ curve in Fig. 3(a) and will fall radially out of the flux tube, i.e., RUM. Since $\lambda=2 B_{\phi} / B_{z} r$, Eq. (6) is identical to Eq. (1). All magnetic flux tubes have uniform $B_{z}$ and $J_{z}$ near the axis so particles with $S<0$ will always be on a potential hill and experience RUM. Equation (6) can be written in terms of experimental parameters as

$$
S=K I v_{z 0} / q+0.5<0,
$$

where $K=\mu_{0} \pi m r^{2} / \Phi^{2}$ is positive, showing that RUM requires $I v_{z 0} / q<0$, i.e., countercurrent flow. Because $K \propto m$, ions have a much lower threshold for RUM than electrons.

Figure 3(a) plots $V(\xi)$ given by Eq. (5) for $S=-0.5$ and 1.5, while Fig. 3(b) plots trajectories calculated from direct numerical integration of $m \ddot{\mathbf{r}}=q \mathbf{v} \times\left(B_{\phi} \hat{\phi}+B_{z} \hat{z}\right)$ for a particle with $S=-0.5$ (i.e., $v_{z 0}=-\omega_{c} / \lambda$ ) starting at the down arrow and for a particle with $S=1.5$ (i.e., $v_{z 0}=$ $\left.+\omega_{c} / \lambda\right)$ starting at the up arrow. Injection at $\lambda x=\lambda y=$ $10^{-6}$ is used so a particle does not start exactly at the top of a potential hill. To approximate the weak field to the right of the flux loop sketched in Fig. 2(b), an exponentially decaying $B_{z}$ in the current-free external region is used in the numerical calculation. Figure 3(b) shows that the $S=$ -0.5 particle is ejected from the flux tube (i.e., RUM), whereas the $S=1.5$ particle remains on the flux tube axis (i.e., is confined).
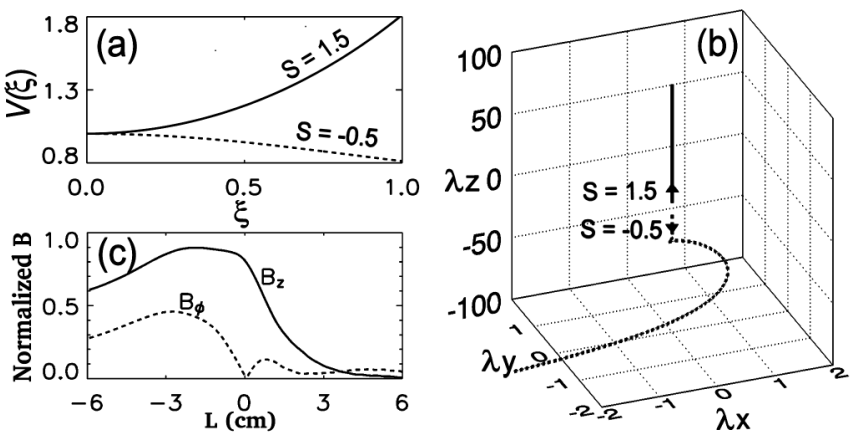

FIG. 3. (a) $S=1.5$ gives "valley" (i.e., stable) effective potential $V(\xi)$ while $S=-0.5$ gives "hill" (i.e., unstable). (b) Numerically calculated particle trajectories. (c) Probe measurement of flux tube magnetic field showing that field is weak on right side as sketched in Fig. 2(b).

Our experimental configuration [12], sketched in Fig. 2(b), involves top and bottom electrodes (respectively, cathode and anode) mounted on the end dome of a large vacuum chamber (base pressure $\sim 10^{-7} \mathrm{mbar}$ ). The experimental sequence is (i) slow ( $\sim 10 \mathrm{~ms})$ electromagnets behind the electrodes create an initial arched vacuum magnetic field, (ii) a fast $(\sim 1 \mathrm{~ms})$ gas valve injects neutral gas from orifices in the electrodes, (iii) a $\sim 1 \mathrm{~kJ}, 59 \mu \mathrm{F}$ capacitor switched across the electrodes breaks down the neutral gas, (iv) a bright plasma loop appears. The 10-20 $\mu$ s dynamical evolution of this loop is imaged [13] by a fast digital framing camera. Detailed measurements in a similar experiment [14] showed that the bulk plasma in the flux loop is many orders of magnitude denser than the injected prebreakdown neutral gas and results from fast MHD ingestion into the loop of orificeoriginating plasma [15]. Figure 3(c) shows magnetic probe [16] measurements of flux tube $B_{\phi}$ and $B_{z}$ as functions of distance $L$ from the flux tube axis in the direction away from the electrode plane (data deconvolved as in Ref. [4]); the magnetic field amplitude decays rapidly to the right [corresponding to weak field region in Fig. 2(b)].

Figure 4 shows the evolution of Ar plasma loops for different injected gas mass $M_{n}$ and different flux $\Phi$ as a function of time measured from breakdown. $M_{n}$ was determined using a thermocouple gauge and has only a relative meaning because the plasma shot, being much shorter than the gas puff time, uses only a fraction of $M_{n}$. Since the plasma has very low impedance, the capacitor acts approximately as a current source. This is consistent with the observation that $I$ and hence $\lambda / \omega_{c}$ are essentially unaffected when $M_{n}$ is varied. However, the plasma velocity $v_{z 0}$ is observed to be strongly dependent on $M_{n}$ with higher $v_{z 0}$ observed at smaller $M_{n}$.

Figure 4(a) corresponds to $M_{n}=4.9 \mathrm{mg}, \quad \Phi=$ $0.75 \mathrm{mWb}$; Fig. 4(b) to $M_{n}=2.3 \mathrm{mg}, \Phi=0.75 \mathrm{mWb}$; and Fig. 4(c) to $M_{n}=2.3 \mathrm{mg}, \Phi=0.25 \mathrm{mWb}$. In the first two frames of Figs. 4(a)-4(c) the plasma has a smooth arch shape; $I$ is low at this stage and the plasma follows the 


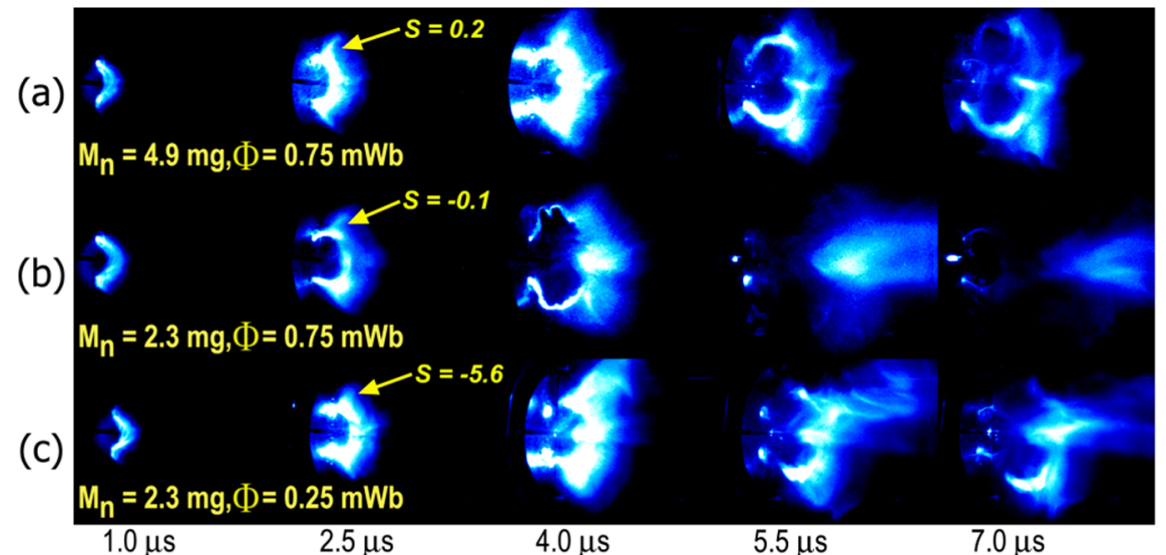

FIG. 4 (color online). Evolution of laboratory plasma loops at (a) high $M_{n}$, (b) low $M_{n}$, and (c) low $\Phi$. Movies placed in Ref. [13] show the evolutions even more dramatically.

half-torus profile of the initial vacuum magnetic field spanning the electrodes. Then, as $I$ increases, the plasma minor radius decreases due to self-pinching while the major radius increases due to the hoop force [17] associated with the poloidal magnetic field produced by $I$. While this is happening, the loop undergoes MHD kink instability and the projection of the writhed loop axis results in a cusplike dip at the apex [17]. In the second frame (i.e., $2.5 \mu \mathrm{s}$ ) of Figs. 4(a) and 4(b) a fingerlike stream of plasma emerges near the top (i.e., near cathode) of the loops. In Fig. 4(b), which corresponds to low $M_{n}$ and hence high $v_{z 0}$, the stream moves toward the ground plane near the cathode and leads to a major disruption in $I$. As also seen in Fig. 4(b), this is followed by the detachment of the loop from the electrodes and, for $t>4 \mu \mathrm{s}$, formation of a plasma jet propagating far to the right of the electrodes (see also Fig. 1) into the weak field region [i.e., to the right in Figs. 2(b) and 3(c)]. A significant drop in $I$ is observed during the detachment phase as well as an associated upward voltage spike. From this time on, $I$ commutates to a new shorter path between the electrodes, while the detached plasma jet propagates away from the electrodes. When $\Phi$ is lowered as shown in Fig. 4(c), two critical stages of the detachment are clearly seen in the 4.0-7.0 $\mu \mathrm{s}$ frames, specifically the loop first detaches from the cathode and then from the anode to form an intense plasma jet. Figures 4(a)-4(c) also display $S$ estimated using measured cathode region quantities in Eq. (7) at $2.5 \mu$ s (i.e., just before detachment) and indicate that the plasma jet development in Figs. 4(b) and 4(c) is associated with having $S<0$.

Equation (7) shows that only ions with $v_{z 0}$ being large and negative relative to $I$ can have $S<0$. Measurements (discussed below) indicate that near the cathode ions with large negative $v_{z 0}(\sim 40-60 \mathrm{~km} / \mathrm{s})$ indeed exist. The slowing-down time $(>100 \mu \mathrm{s})$ of these fast ions by the plasma (density $\sim 10^{20} \mathrm{~m}^{-3}$ ) is much longer than the plasma duration; therefore, collisions cannot affect their orbits. Since the ion contribution to electric current necessarily flows in the same direction as the current, ion drift motion associated with electric current cannot account for the observed large negative $v_{z 0}$. Furthermore, because the measured $\left|v_{z 0}\right|$ greatly exceeds the $\mathrm{Ar}^{+}$thermal speed $v_{T} \sim 2-5 \mathrm{~km} / \mathrm{s}$ estimated using the spectroscopically determined $T_{i} \sim 1-10 \mathrm{eV}$, neither can ion thermal motion account for the observed large negative $v_{z 0}$. However, there does exist a mechanism capable of accelerating ions to high velocities either parallel or antiparallel to $I$. This mechanism [14,15] shows that axial gradients of $B_{\phi}^{2}$ provide an MHD force $\sim-\partial B_{\phi}^{2} / \partial z$ that accelerates plasma from regions of large $B_{\phi}^{2}$ to regions of small $B_{\phi}^{2}$; i.e., acceleration occurs from both foot-points of a flux loop towards the apex if the flux loop minor radius is smaller at the foot-points than at the apex (see detailed discussion in Ref. [15]). The resulting velocity is $v_{z} \sim B_{\phi} / \sqrt{\mu_{0} m_{i} n_{i}}$, consistent with higher ion axial velocity observed at smaller neutral gas injection pressures.

$\mathrm{Ar}^{+}$Doppler velocity measurements have been made using a $1 \mathrm{~m}$ monochromator with a gated intensified CCD camera with fiber and lens coupling system. The spectra displayed in Fig. 5 show velocity components along lines of sight (los) indicated in Fig. 1 by "los \#". The los \#1 and \#2 spectra in Fig. 5(a) show that both cathode and anode emission lines are blueshifted, confirming suprathermal ion flow from both cathode and anode towards the apex as predicted by Ref. [15]. This outflow is seen in camera
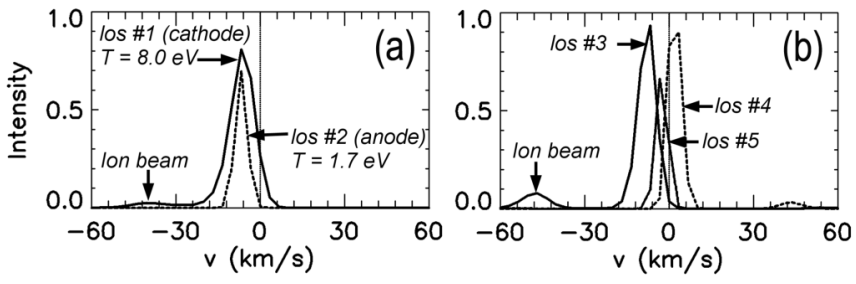

FIG. 5. Measured spectra of an $\mathrm{Ar}^{+}$line (rest-frame wavelength $\lambda_{0}=434.806 \mathrm{~nm}$ shown by vertical lines). Velocity is $v=c\left(\lambda-\lambda_{0}\right) / \lambda_{0}$ where $\lambda$ is measurement wavelength, $c$ is speed of light. Lines of sight (los \#) are shown in Fig. 1. (a) Spectra from the cathode (los \#1) and anode (los \#2) regions for $t=0-0.5 \mu \mathrm{s}$. (b) Spectra from the kinetic jet region along los \#3, \#4, and \#5 for $t=2.5-5.5 \mu \mathrm{s}, 7.0-9.0 \mu \mathrm{s}$, and 6.0-18.0 $\mu \mathrm{s}$, respectively. 


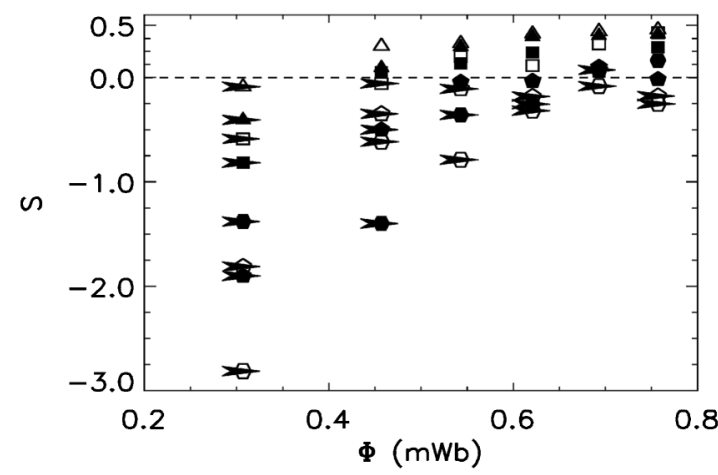

FIG. 6. Measured $S$ for various plasma configurations. Solid (open) symbols represent high (low) $M_{n}$ respectively, number of sides in symbols represent capacitor charging voltage in $\mathrm{kV}$, and arrowheads indicate plasma shots where kinetic jets are observed. The existence of kinetic jets has an excellent correlation with $S$ being negative.

images as $30-60 \mathrm{~km} / \mathrm{s}$ bright fronts propagating away from both electrodes along the flux loop axis towards its apex [13]. Figure 5(a) shows a large ion velocity component $(\sim 40 \mathrm{~km} / \mathrm{s}$ ion beam for los \#1) moving away from the cathode while Fig. 5(b) shows spectra measured in the kinetic jet region and indicates a $\sim 50 \mathrm{~km} / \mathrm{s}$ ion beam for los \#3. Because, as seen in Fig. 1, los \#1 and los \#3 make different angles relative to the respective flow directions being measured, ion beam velocities between different los \#'s cannot be quantitatively compared; i.e., the $50 \mathrm{~km} / \mathrm{s}$ los \#3 ion beam in Fig. 5(b) cannot be interpreted as a $10 \mathrm{~km} / \mathrm{s}$ acceleration of the $40 \mathrm{~km} / \mathrm{s}$ los \#1 ion beam in Fig. 5(a).

Figure 6 shows the results of a parameter scan of $I, \Phi$, and $v_{z 0}$ performed to determine the $S$ dependence of the instability onset. $v_{z 0}$ is determined from plasma front motion in the camera images [13]. The $S$ values in Fig. 6 were calculated using cathode region parameters in Eq. (7) for a large number of argon plasma loops $(r \simeq 8 \mathrm{~mm}) ; S=$ 0.5 is the upper bound for the observed negative $I v_{z 0}$. Kinetic jet instability, shown by arrowheads in Fig. 6, occurs only when $S<0$ indicating excellent agreement with the RUM onset prediction.

The plasma loops used for Fig. 6 have already undergone MHD kink instability [18] since all have $|\lambda|>$ $4 \pi / L \simeq 60 \mathrm{~m}^{-1}$, where $L \simeq 0.2 \mathrm{~m}$ is the loop length. The loops produce kinetic jets only when $S<0$ showing that RUM is a kinetic, rather than MHD, instability. The kinetic nature is also evident from the high velocity beams in Fig. 5 and from the kinetic jet appearing in the weak field (non-MHD) region as sketched in Fig. 2(b).

The RUM model explains why counterinjected neutral beams in tokamaks have severe orbit losses compared to coinjected neutral beams [5-7]. In particular, Fig. 10 of Ref. [5] showed that an $80 \mathrm{keV}$ counterinjected deuterium beam has severe orbit losses in a $B=0.3 \mathrm{~T}$ tokamak having safety factor $q=1.25$ and major radius $R \simeq 1 \mathrm{~m}$.
Since $\lambda \simeq 2 / q R \simeq 1.6 \mathrm{~m}^{-1}, \omega_{c D}=1.4 \times 10^{7} \mathrm{~s}^{-1}$, and the injection velocity is $v_{\text {inj }}=2.8 \times 10^{6} \mathrm{~m} / \mathrm{s}$, it is seen that $S_{\text {counter }} \simeq 0.5-\lambda v_{\text {inj }} / \omega_{c D} \approx 0.2$ whereas $S_{c o}=0.5+$ $\lambda v_{\text {inj }} / \omega_{c D}=0.8$; so, counterinjected ions have much larger orbits ([i.e., broader valley-type effective potential as in Fig. 3(a)] than coinjected ions. While coronal loops are unlikely to have $S<0$ due to their small $\lambda\left(\sim 10^{-8} \mathrm{~m}^{-1}\right)$ [19], jets associated with canceling magnetic features [8-10] and coronal streamers [11] emanating near magnetic neutral lines are both produced in extremely low magnetic field regions where $S<0$ could occur and RUM may be operative.

In summary, an instability has been demonstrated where ions are magnetically ejected from a flux tube. Ejection occurs when ions move opposite to the current with a sufficiently large axial velocity.

We thank A.H. Boozer for pointing out a relationship between RUM and neutral beam counterinjection and D. Felt for technical assistance. Supported by U.S. DOE and by NSF.

*Present address: Physics and Astronomy, University of California, Los Angeles, Los Angeles, CA 90095, USA.

[1] E. Tandberg-Hanssen, The Nature of Solar Prominences (Kluwer Academic, Dordrecht, 1995).

[2] P. M. Bellan, Spheromaks (Imperial College Press, London, 2000).

[3] J. Sheffield, Rev. Mod. Phys. 66, 1015 (1994).

[4] L. F. Burlaga, J. Geophys. Res. 93, 7217 (1988).

[5] D. R. Mikkelsen et al., Phys. Plasmas 4, 3667 (1997).

[6] J. Egedal et al., Phys. Plasmas 10, 2372 (2003).

[7] K. G. McClements and A. Thyagaraja, Phys. Plasmas 13, 042503 (2006).

[8] J. Chae, Astrophys. J. 584, 1084 (2003).

[9] Y. Liu and H. Kurokawa, Astrophys. J. 610, 1136 (2004).

[10] R. A. Harrison, P. Bryans, and R. Bingham, Astron. Astrophys. 379, 324 (2001).

[11] J. Li et al., Astrophys. J. 506, 431 (1998).

[12] J.F. Hansen, S. K.P. Tripathi, and P. M. Bellan, Phys. Plasmas 11, 3177 (2004).

[13] See EPAPS Document No. E-PRLTAO-98-097714 for movies and camera $v_{z 0}$ measurement example. For more information on EPAPS, see http://www.aip.org/pubservs/ epaps.html.

[14] S. You, G. S. Yun, and P. M. Bellan, Phys. Rev. Lett. 95, 045002 (2005).

[15] P. M. Bellan, Phys. Plasmas 10, 1999 (2003).

[16] C. A. Romero-Talamas, P. M. Bellan, and S. C. Hsu, Rev. Sci. Instrum. 75, 2664 (2004).

[17] J.F. Hansen and P. M. Bellan, Astrophys. J. 563, L183 (2001).

[18] S. C. Hsu and P. M. Bellan, Phys. Rev. Lett. 90, 215002 (2003).

[19] A. B. Burnette and R. C. Canfield, Astrophys. J. 606, 565 (2004). 